\title{
Investigations on the EPR Parameters of $\mathrm{KMgF}_{3}: \mathrm{Cr}^{+}$
}

\author{
M.Q. KuAnG, S.Y. Wu, X.F. Hu, G.L. Li And H.Y. Zu \\ School of Physical Electronics, University of Electronic Science and Technology of China \\ Chengdu 610054, P.R. China
}

(Received May 24, 2013; in final form March 13, 2014)

\begin{abstract}
The electron paramagnetic resonance parameters (i.e., $g$ factor, hyperfine structure constant and superhyperfine parameters) of $\mathrm{KMgF}_{3}: \mathrm{Cr}^{+}$are theoretically investigated from the perturbation formulae of these parameters for an octahedral $3 d^{5}$ cluster. As for the calculations of $g$ factor and hyperfine structure constant, both the contributions from the crystal-field and charge transfer mechanisms are included based on the cluster approach. The metal to ligand charge transfer contribution to the $g$-shift $\Delta g(\approx g-2.0023)$ is the same (negative) in sign and much larger in magnitude as compared to the crystal-field one. The conventional argument that the charge transfer contributions to zero-field splittings are negligible for $3 d^{5}$ ions in fluorides is no longer suitable for $\Delta g$ analysis of $\mathrm{KMgF}_{3}: \mathrm{Cr}^{+}$due to the dominant second-order charge transfer perturbation term. The charge transfer contribution to hyperfine structure constant exhibits the same sign and about $4 \%$ of the crystal-field one. The unpaired spin densities of the fluorine $2 s, 2 p \sigma$ and $2 p \pi$ orbitals are quantitatively acquired from the relationships with the relevant molecular orbital coefficients using the uniform model. The present treatments are superior to the previous calculations of directly fitting the experimental superhyperfine parameters.
\end{abstract}

DOI: 10.12693/APhysPolA.125.1224

PACS: $75.10 . \mathrm{Dg}, 76.30 . \mathrm{Fc}$

\section{Introduction}

Fluoroperovskite $\mathrm{KMgF}_{3}$ containing chromium show unique luminescence [1], thermoluminescence [2], optical [3], thermal conductivity [4], and other physical properties [5] as well as promising applications in optical components [6] and ceramic science and technology [7]. Normally, the above properties may be closely correlated to the structure and electronic properties of the Cr dopants in the host materials, which may be conveniently investigated by means of the electron paramagnetic resonance (EPR) and electron nuclear double resonance (ENDOR) techniques. On the other hand, $3 d^{5}$ configuration is usually regarded as a model system in the transition-metal group due to the half-filled $3 d$ sub-shell and the orbital non-degenerate ${ }^{6} A_{1 \mathrm{~g}}$ ground state of high spin $S=5 / 2$ [8-10]. Unlike more conventional $\mathrm{Mn}^{2+}$ and $\mathrm{Fe}^{3+}, \mathrm{Cr}^{+}\left(3 d^{5}\right)$ is relatively scarce. It is also rare as compared to various high valence states $(+n$, with $n=2-5$ ) of chromium and seldom studied. So, studies on $\mathrm{Cr}^{+}$in crystals would be of scientific and practical importance. For example, the EPR and ENDOR experiments were carried out for $\mathrm{Cr}^{+}$doped $\mathrm{KMgF}_{3}$, and the EPR parameters ( $g$ factor, hyperfine structure constant $A$ and superhyperfine parameters $A^{\prime}$ and $B^{\prime}$ ) were measured for ${ }^{53} \mathrm{Cr}^{+}-\mathrm{F}^{-}$combination $[11,12]$.

Until now, however, these experimental results have not been theoretically explained, only the superhyperfine parameters were tentatively analyzed by directly fitting the unpaired spin densities to the experimental superhyperfine parameters [13]. So, the obtained unpaired spin densities $f_{\mathrm{s}}$ and $f_{\sigma}-f_{\pi}$ were not quantitatively correlated with chemical bonding between the central ion and ligands but simply taken as the adjustable parameters. Moreover, no uniform theoretical investigations for the $g$ factor and the hyperfine structure constant have been made yet. In order to overcome the above shortcomings of the previous studies [13] and to study the EPR spectra for $\mathrm{KMgF}_{3}: \mathrm{Cr}^{+}$to a better extent, further theoretical analyses of the EPR parameters are of significance. It is noted that not only the crystal-field mechanism related to the antibonding orbitals but also the charge transfer mechanism related to the bonding (and non-bonding) orbitals may induce contributions to the EPR parameters [14]. Because of the very small $\left(\approx 10^{-3}[11]\right) g$-shift $\Delta g\left(\approx g-g_{\mathrm{s}}\right.$, where $g_{\mathrm{s}} \approx 2.0023$ is the spin-only value $)$ for $\mathrm{KMgF}_{3}: \mathrm{Cr}^{+}$due to the non-degenerate ${ }^{6} A_{1 \mathrm{~g}}$ ground state, the charge transfer mechanism can amount obvious importance in $\Delta g$ despite of weak covalency.

In this work, the improved perturbation formulae of the EPR parameters are adopted for an octahedral $3 d^{5}$ cluster. In the treatments of $g$ factor and hyperfine structure constant, both the crystal-field and charge transfer contributions are uniformly included from the cluster approach. As for superhyperfine parameters, the unpaired spin densities for the fluorine $2 s$ and $2 p$ orbitals are quantitatively acquired using the theoretical formulae associated with the relevant molecular orbital coefficients from the cluster approach. The results are discussed.

\section{Theoretical formulae and calculations}

According to Ref. [13], cubic point symmetry can be maintained when $\mathrm{Mg}^{2+}$ in $\mathrm{KMgF}_{3}$ is replaced by a divalent iron-group impurity. In particular, chromium would dissolve almost entirely in the divalent state and yield $\mathrm{Cr}^{+}$and $\mathrm{Cr}^{3+}$ on cubic $\mathrm{Mg}^{2+}$ sites with nearly equal concentrations under X-ray irradiation at room temperature. On the other hand, the probability of charge compensation (e.g., $\mathrm{O}^{2-}$ or $\mathrm{K}^{+}$vacancies for $\mathrm{Cr}^{+}$or $\mathrm{Cr}^{3+}$ substitution) would be small at very low temperature $(4 \mathrm{~K})$ 
of ENDOR measurements [11, 12] and can be neglected. So, $\mathrm{Cr}^{+}$may occupy the host $\mathrm{Mg}^{2+}$ site and conserve original $O_{h}$ symmetry in $\mathrm{KMgF}_{3}$. For a $3 d^{5}$ ion under a perfect octahedron, the system shows the orbital non-degenerate ${ }^{6} A_{1 \mathrm{~g}}$ ground state with high spin $S=5 / 2$ $[8,9]$. According to the extensive EPR investigations for $3 d^{5}$ ions in compounds, combination of the spin-orbit coupling and orbital angular momentum interactions is normally regarded as the dominant origin of $g$-shift $\Delta g$ and zero-field splittings $[15,16]$. Importantly, the metal to ligand charge transfer contribution is expected due to the low valence state of $\mathrm{Cr}^{+}$in the $\mathrm{Cr}^{+}-\mathrm{F}^{-}$combination, and this contribution may be significant because of the delicious $\Delta g$ and should be taken into account in the EPR studies for $\mathrm{KMgF}_{3}: \mathrm{Cr}^{+}$.

\subsection{The perturbation formulae of $g$ factor and hyperfine structure constant}

For the studied system the metal to ligand charge transfer mechanism should be considered. Thus, the nine-electron wave functions containing the anti-bonding orbitals $e^{a}$, the non-bonding orbitals $t_{2}^{n}$ and the bonding orbitals $e^{b}$ are adopted here. The ground state ${ }^{6} A_{1 \mathrm{~g}}$ is expressed as follows:

$$
\left|{ }^{6} A_{1} \frac{5}{2} a_{1}\right\rangle=\left[\xi^{+} \eta^{+} \zeta^{+} \theta^{+} \varepsilon^{+} \mid \theta^{+} \theta^{t} \varepsilon^{+} \varepsilon^{-}\right] .
$$

In the square bracket on the right hand of the above expression, the letters $(\xi, \eta, \zeta$ and $\theta, \varepsilon)$ in the left column are the $t_{2}^{n}$ and $e^{a}$ orbitals and those $(\theta$ and $\varepsilon)$ in the right column are the $e^{b}$ ones. For the metal to ligand charge transfer, there is only one excited state $\left(t_{2}^{n}\right)^{3}\left(e^{a}\right)^{1}\left(t_{2}^{b}\right)^{1}\left(e^{b}\right)^{4}$ (or ${ }^{6} T_{1}^{n}$ ) having non-zero spin-orbit coupling interaction with the ground ${ }^{6} A_{1 \mathrm{~g}}$ state. The $z$ component of the charge transfer excited state ${ }^{6} T_{1}^{n}$ with the highest $M_{\mathrm{S}}=5 / 2$ can be expressed as

$$
\left|{ }^{6} T_{1}^{n} \frac{5}{2} z\right\rangle=\left[\xi^{+} \eta^{+} \zeta^{+} \theta^{+} \mid \zeta^{+} \theta^{+} \theta^{-} \varepsilon^{+} \varepsilon^{-}\right] .
$$

From the cluster approach [17], the one-electron basis functions for an octahedral $3 d^{5}$ cluster may be written in terms of the LCAO-MO orbitals

$$
\begin{aligned}
& \psi_{t}^{x}=\left(N_{t}^{x}\right)^{1 / 2}\left(\varphi_{t}-\lambda_{t}^{x} \chi_{p t}\right), \\
& \psi_{e}^{x}=\left(N_{e}^{x}\right)^{1 / 2}\left(\varphi_{e}-\lambda_{e}^{x} \chi_{p e}-\lambda_{s}^{x} \chi_{s}\right) .
\end{aligned}
$$

Here the superscript $x$ (=a and $b)$ stand for the anti-bonding and bonding orbitals, respectively. $\varphi_{\gamma}(\gamma=e$ and $t$ denote the irreducible representations $E_{\mathrm{g}}$ and $T_{2 \mathrm{~g}}$ of the $O_{h}$ group) are the pure impurity $3 d$ orbitals. $\chi_{p \gamma}$ and $\chi_{s}$ are the pure fluorine $2 p$-and $2 s$-orbitals. $N_{\gamma}$ and $\lambda_{\gamma}\left(\right.$ or $\left.\lambda_{s}\right)$ are, respectively, the normalization factors and the orbital admixture coefficients.

Utilizing the perturbation procedure [16], the improved formulae for $g$ factor and hyperfine structure constant can be obtained as follows:

$$
\begin{aligned}
& \Delta g=\Delta g_{\mathrm{CF}}+\Delta g_{\mathrm{CT}}, \\
& \Delta g_{\mathrm{CF}}=-5 \zeta_{\mathrm{CF}}^{\prime 2}\left(1 / E_{1}^{2}+1 / E_{3}^{2}\right) / 6-\zeta_{\mathrm{CF}}^{2} / E_{2}^{2} \\
& \quad-8 \zeta_{\mathrm{CF}}^{\prime} \zeta_{\mathrm{CF}}\left[1 /\left(E_{1} E_{2}\right)+1 /\left(E_{2} E_{3}\right)\right], \\
& \Delta g_{\mathrm{CT}}=-8 \zeta_{\mathrm{CT}}^{\prime} k_{\mathrm{CT}}^{\prime} /\left(5 E_{n}\right),
\end{aligned}
$$

$$
\begin{aligned}
& A=A_{\mathrm{CF}}+A_{\mathrm{CT}}, \\
& A_{\mathrm{CF}}=-P_{\mathrm{CF}}^{\prime}\left\{5 \zeta_{\mathrm{CF}}^{\prime 2}\left(1 / E_{1}^{2}+1 / E_{3}^{2}\right) / 6+\zeta_{\mathrm{CF}}^{2} / E_{2}^{2}\right. \\
& \left.\quad+8 \zeta_{\mathrm{CF}}^{\prime} \zeta_{\mathrm{CF}}\left[1 /\left(E_{1} E_{2}\right)+1 /\left(E_{2} E_{3}\right)\right]\right\}-\kappa P_{\mathrm{CF}}, \\
& A_{\mathrm{CT}}=8 P_{\mathrm{CT}}^{\prime} k_{\mathrm{CT}}^{\prime} \zeta_{\mathrm{CT}}^{\prime} /\left(5 E_{n}\right)-\kappa P_{\mathrm{CT}} / 4 .
\end{aligned}
$$

In the above expressions, the denominators $E_{i}(i=$ $1-3)$ are the energy separations between the crystal-field excited ${ }^{4} T_{1 \mathrm{~g}},{ }^{4} T_{2 \mathrm{~g}}$ and ${ }^{2} T_{2 \mathrm{~g}}$ and the ground ${ }^{6} A_{1 \mathrm{~g}}$ states. They are normally obtained in terms of the cubic field parameter $\mathrm{Dq}$ and the Racah parameters $B$ and $C$ for the $3 d^{5}$ ion in crystals $[18,19]$ :

$$
\begin{aligned}
& E_{1} \approx 10 B+6 C-10 D q, \\
& E_{2} \approx 19 B+7 C, \\
& E_{3} \approx 10 B+6 C+10 D q .
\end{aligned}
$$

Here, $\zeta_{\mathrm{CF}}, \zeta_{\mathrm{CT}}, \zeta_{\mathrm{CF}}^{\prime}$ and $\zeta_{\mathrm{CT}}^{\prime}$ are the spin-orbit coupling coefficients, $k_{\mathrm{CT}}^{\prime}$ is the orbital reduction factor, and $P_{\mathrm{CF}}, P_{\mathrm{CF}}^{\prime}, P_{\mathrm{CT}}$ and $P_{\mathrm{CT}}^{\prime}$ are the dipolar hyperfine structure parameters for the $3 d^{5}$ ion in crystals. The subscripts CF and CT stand for the corresponding interactions related to the crystal-field and charge transfer mechanisms, respectively. $E_{n}$ is the energy difference between the charge transfer excited ${ }^{6} T_{1}^{n}$ and the ground ${ }^{6} A_{1 \mathrm{~g}}$ states.

Utilizing the cluster approach [17], the spin-orbit coupling coefficients and the dipolar hyperfine structure parameters for the crystal-field mechanism can be expressed as follows:

$$
\begin{aligned}
& \zeta_{\mathrm{CF}}=N_{t}^{a}\left[\zeta_{d}^{0}+\left(\lambda_{t}^{a}\right)^{2} \zeta_{p}^{0} / 2\right], \\
& \zeta_{\mathrm{CF}}^{\prime}=\left(N_{t}^{a} N_{e}^{a}\right)^{1 / 2}\left[\zeta_{d}^{0}-\lambda_{t}^{a} \lambda_{e}^{a} \zeta_{p}^{0} / 2\right], \\
& P_{\mathrm{CF}}=N_{t}^{a} P_{0}, \\
& P_{\mathrm{CF}}^{\prime}=\left(N_{t}^{a} N_{e}^{a}\right)^{1 / 2} P_{0} .
\end{aligned}
$$

Similarly, those for the charge transfer mechanism are

$$
\begin{aligned}
& \zeta_{\mathrm{CT}}^{\prime}=\left(N_{t}^{a} N_{e}^{b}\right)^{1 / 2}\left[\left(1+\lambda_{t}^{a}-\lambda_{s}^{a}\right) \zeta_{d}^{0}-\lambda_{t}^{a} \lambda_{e}^{a} \zeta_{p}^{0} / 2\right], \\
& k_{\mathrm{CT}}^{\prime}=\left(N_{t}^{a} N_{e}^{b}\right)^{1 / 2}\left[1-\lambda_{e}^{a}+\lambda_{t}^{a}-2 \lambda_{t}^{a} S_{t} S_{e}\right. \\
& \left.\quad+\lambda_{e}^{a} \lambda_{t}^{a} S_{t} / 2+A \lambda_{t}^{b} \lambda_{s}^{a} / 2\right], \\
& P_{\mathrm{CT}}=N_{t}^{b} P_{0}, \\
& P_{\mathrm{CT}}^{\prime}=\left(N_{t}^{b} N_{e}^{b}\right)^{1 / 2} P_{0} .
\end{aligned}
$$

In the above formulae, $\zeta_{d}^{0}$ and $\zeta_{p}^{0}$ are, respectively, the spin-orbit coupling coefficients of the free $3 d^{5}$ and ligand ions. $P_{0}$ is the dipolar hyperfine structure parameter of the free $3 d^{5}$ ion. $A$ denotes the integral $R\left\langle\chi_{s}|\partial / \partial x| \chi_{p x}\right\rangle$ between the ligand $2 s$ and $2 p$ orbitals, with the impurityligand (reference) distance $R$.

The molecular orbital coefficients in Eq. (3) can be determined from the cluster approach [17]. One can obtain the normalization conditions

$$
\begin{aligned}
& N_{t}^{x}\left[1+\left(\lambda_{t}^{x}\right)^{2}-2 \lambda_{t}^{x} S_{t}\right]=1, \\
& N_{e}^{x}\left[1+\left(\lambda_{e}^{x}\right)^{2}+\left(\lambda_{s}^{x}\right)^{2}-2 \lambda_{e}^{x} S_{e}-2 \lambda_{s}^{x} S_{s}\right]=1
\end{aligned}
$$

and the orthogonality relationships

$$
\begin{aligned}
& 1+\lambda_{t}^{a} \lambda_{t}^{b}-\left(\lambda_{t}^{a}+\lambda_{t}^{b}\right) S_{t}=0, \\
& 1+\lambda_{e}^{a} \lambda_{e}^{b}+\lambda_{s}^{a} \lambda_{s}^{b}-\left(\lambda_{e}^{a}+\lambda_{e}^{b}\right) S_{e}-\left(\lambda_{s}^{a}+\lambda_{s}^{b}\right) S_{s}=0, \\
& \lambda_{e}^{a} \lambda_{s}^{b}+\lambda_{s}^{a} \lambda_{e}^{b}=0 .
\end{aligned}
$$

Here $S_{t}, S_{e}$ and $S_{s}$ are the group overlap integrals be- 
tween the impurity $3 d$ and the ligand $2 p$ and $2 s$ orbitals. The following approximation relationships are satisfied for the antibonding orbitals [17]:

$$
\begin{aligned}
& N^{2} \approx\left(N_{t}^{a}\right)^{2}\left[1+\left(\lambda_{t}^{a}\right)^{2} S_{t}^{2}-2 \lambda_{t}^{a} S_{t}\right], \\
& N^{2} \approx\left(N_{e}^{a}\right)^{2}\left[1+\left(\lambda_{e}^{a}\right)^{2} S_{e}^{2}+\left(\lambda_{s}^{a}\right)^{2} S_{s}^{2}-2 \lambda_{e}^{a} S_{e}-2 \lambda_{s}^{a} S_{s}\right] .
\end{aligned}
$$

In these formulae, $N$ denotes the average covalency factor, characteristic of covalency of the system. Since the orbital admixture coefficients decrease with decrease of the group overlap integrals as the distance $R$ increases, the proportional relationship $\lambda_{s} / \lambda_{e} \approx S_{s} / S_{e}$ can be approximately adopted for the orbital admixture coefficients and the related group overlap integrals within the same $E_{\mathrm{g}}$ irreducible representation.

\subsection{The formulae of superhyperfine parameters}

It is noted that the unpaired spin densities $f_{s}$ and $f_{\sigma}-f_{\pi}$ of the ligand $\mathrm{F}^{-} 2 s$ and $2 p \sigma$ (or $2 p \pi$ ) orbitals were usually treated as adjustable parameters in the previous calculations [13], instead of being quantitatively connected with chemical bonding of the central ion and ligands. However, these quantities are determined from the uniform theoretical formulae based on the cluster approach [17]. The superhyperfine parameters are expressed as

$$
\begin{aligned}
& A^{\prime}=A_{\mathrm{s}}+2\left(A_{\mathrm{D}}+A_{\sigma}-A_{\pi}\right), \\
& B^{\prime}=A_{\mathrm{s}}-\left(A_{\mathrm{D}}+A_{\sigma}-A_{\pi}\right) .
\end{aligned}
$$

Here $A_{\mathrm{s}}$ is the isotropic contribution to the superhyperfine parameters, characteristic of the influence of the ligand $2 s$ orbital. $A_{\mathrm{D}}$ and $A_{\sigma}-A_{\pi}$ stand for the anisotropic contributions from the dipole-dipole interaction between the electron of the central ion and ligand nucleus and that from the ligand $2 p$ orbital, respectively. The isotropic part can be further expressed as follows [20]:

$$
A_{\mathrm{s}}=f_{\mathrm{s}} A_{\mathrm{s}}^{0} /(2 S) \text {. }
$$

Here $A_{\mathrm{s}}^{0}=(8 / 3) g_{s} g_{n} \beta \beta_{n}|\Psi(0)|^{2} \approx 15000 \times 10^{-4} \mathrm{~cm}^{-1}$ and $A_{\mathrm{p}}^{0}=g_{s} g_{n} \beta \beta_{n}\left\langle r^{-3}\right\rangle_{2 p} \approx 1072 \times 10^{-4} \mathrm{~cm}^{-1}$ [21] for the ligand $\mathrm{F}^{-}$.

The electron spin is $S=5 / 2$ of the ground state ${ }^{6} A_{1 \mathrm{~g}}$ for $\mathrm{Cr}^{+}$in $\mathrm{KMgF}_{3} . f_{s}$ stands for the unpaired spin density of the ligand $2 s$ orbital. The anisotropic contribution from the fluorine $2 p$ orbital is often expressed as [20]:

$$
A_{\sigma}-A_{\pi}=A_{\mathrm{p}}^{0}\left(f_{\sigma}-f_{\pi}\right) /(2 S) .
$$

Here $f_{\sigma}$ and $f_{\pi}$ are the unpaired spin densities of the ligand $2 p \sigma$ and $2 p \pi$ orbitals, respectively. The dipoledipole interaction between the electron distribution of the central ion and the fluorine nucleus can be written as $A_{\mathrm{D}}=g \beta g_{n} \beta_{n} / R^{3}$, with the $g$ factor of the central ion. The unpaired spin densities are theoretically involved with the related molecular orbital coefficients from the cluster approach

$$
\begin{aligned}
& f_{s} \approx N_{e}^{a}\left(\lambda_{s}^{a}\right)^{2} / 3, \\
& f_{\sigma} \approx N_{e}^{a}\left(\lambda_{e}^{a}\right)^{2} / 3, \\
& f_{\pi} \approx N_{t}^{a}\left(\lambda_{t}^{a}\right)^{2} / 4 .
\end{aligned}
$$

Therefore, the unpaired spin densities are quantitatively determined for the fluorine $2 s, 2 p \sigma$, and $2 p \pi$ orbitals here in a uniform way.

\subsection{Application to $\mathrm{KMgF}_{3}: \mathrm{Cr}^{+}$}

Now these formulae are applied to the investigations of the EPR parameters for $\mathrm{KMgF}_{3}: \mathrm{Cr}^{+}$. Normally, the impurity-ligand distance $R$ in the defect center is dissimilar to the host cation-anion distance $R_{\mathrm{H}}(\approx 1.994 \AA[22])$ due to size or/and charge mismatch of the host $\mathrm{Mg}^{2+}$ by the impurity $\mathrm{Cr}^{+}$[23]. Based on the Hartree-FockRoothann calculations of the ${ }^{6} A_{1 \mathrm{~g}}\left(t_{2 \mathrm{~g}}^{3} e_{\mathrm{g}}^{2}\right)$ ground state for the octahedral $\left[\mathrm{CrF}_{6}\right]^{5-}$ cluster, the equilibrium $\mathrm{Cr}^{+}-\mathrm{F}^{-}$ distance $R$ was found to be about $2.35 \AA$ [23]. From the optical spectra for $3 d^{5}$ ions in fluorides [9], the cubic field parameter $D q \approx 780 \mathrm{~cm}^{-1}$ and the covalency factor $N \approx 0.96$ may be determined. Thus, the Racah parameters $B$ and $C$ of the studied system can be obtained from the free-ion values $B_{0} \approx 830$ and $C_{0} \approx 3430 \mathrm{~cm}^{-1}$ for $\mathrm{Cr}^{+}$[19] using the relationships $B \approx B_{0} N^{2}$ and $C \approx C_{0} N^{2}$ [24]. In view of the charge transfer level $\left(\approx 8900 \mathrm{~cm}^{-1}\right.$ [25]) for $\mathrm{NaCl}: \mathrm{Cr}^{+}$, the value $E_{n} \approx 9300 \mathrm{~cm}^{-1}$ can be estimated for the studied $\mathrm{KMgF}_{3}: \mathrm{Cr}^{+}$from the spectral chemical series $[9,19]$. Utilizing the impurity-ligand distance $R$ and the Slater-type self-consistent field (SCF) wave functions [26, 27], the group overlap integrals are calculated: $S_{t} \approx 0.0056$, $S_{e} \approx 0.0228, S_{s} \approx 0.0183$, and $A \approx 1.7439$. Then the molecular orbital coefficients $N_{\gamma}^{x}$ and $\lambda_{\gamma}^{x}$ are acquired for the antibonding and bonding orbitals from Eqs. (6)-(8).

Utilizing Eqs. (9) and (10) and the free-ion values $\zeta_{d}^{0} \approx$ $230 \mathrm{~cm}^{-1}$ [19] and $P_{0} \approx-29.5 \times 10^{-4} \mathrm{~cm}^{-1}$ [28] for $\mathrm{Cr}^{+}$ and $\zeta_{p}^{0} \approx 220 \mathrm{~cm}^{-1}$ [29] for the ligand $\mathrm{F}^{-}$, the spinorbit coupling coefficients, the orbital reduction factors, and the dipolar hyperfine structure parameters related to the crystal-field and charge transfer mechanisms are determined and given in Table I. The core polarization constant in hyperfine structure constant can be taken as $\kappa \approx 0.7[30]$ for the studied system.

TABLE I

The normalization factors and the orbital admixture coefficients, the spin-orbit coupling coefficients (in $\mathrm{cm}^{-1}$ ), the orbital reduction factors and the dipolar hyperfine structure parameters (in $10^{-4} \mathrm{~cm}^{-1}$ ) related to the crystal-field and charge transfer mechanisms for $\mathrm{KMgF}_{3}: \mathrm{Cr}^{+}$.

\begin{tabular}{c|c|c|c|c|c|c|c|c|c}
\hline \hline$N_{t}^{a}$ & $N_{e}^{a}$ & $N_{t}^{b}$ & $N_{e}^{b}$ & $\lambda_{t}^{a}$ & $\lambda_{e}^{a}$ & $\lambda_{s}^{a}$ & $\lambda_{t}^{b}$ & $\lambda_{e}^{b}$ & $\lambda_{s}^{b}$ \\
\hline 0.959 & 0.965 & 0.147 & 0.159 & 0.210 & 0.173 & 0.162 & -2.407 & -1.744 & -1.407 \\
\hline$\zeta_{\mathrm{CF}}$ & $\zeta_{\mathrm{CF}}^{\prime}$ & $\zeta_{\mathrm{CT}}^{\prime}$ & $k_{\mathrm{CF}}$ & $k_{\mathrm{CF}}^{\prime}$ & $k_{\mathrm{CT}}^{\prime}$ & $P_{\mathrm{CF}}$ & $P_{\mathrm{CF}}^{\prime}$ & $P_{\mathrm{CT}}$ & $P_{\mathrm{CT}}^{\prime}$ \\
\hline 226 & 218 & 100 & 0.982 & 0.968 & 0.303 & -28 & -28 & -4 & -5
\end{tabular}

Substituting these values into Eq. (4), the $g$ factor and the hyperfine structure constant are calculated for $\mathrm{KMgF}_{3}: \mathrm{Cr}^{+}$and shown in Table II. The unpaired spin densities $f_{i}(i=\sigma, \pi, s)$ and hence the isotropic contribution $A_{s}$ and the anisotropic contributions $A_{\sigma}-A_{\pi}$ and $A_{\mathrm{D}}$ to the superhyperfine parameters are calculated from Eqs. (12)-(14). Thus the resultant $A^{\prime}$ and $B^{\prime}$ are calculated from Eq. (11) and given in Table II. In order to 
TABLE II

The $g$-shift $\Delta g$, hyperfine structure constant (in $10^{-4} \mathrm{~cm}^{-1}$ ) and superhyperfine parameters (in $10^{-4} \mathrm{~cm}^{-1}$ ) for $\mathrm{KMgF}_{3}: \mathrm{Cr}^{+}$.

\begin{tabular}{c|c|c|c|c}
\hline \hline & $\Delta g$ & $A$ & $A^{\prime}$ & $B^{\prime}$ \\
\hline calc. $^{a}$ & -0.0010 & 19.943 & 24.3 & 16.8 \\
calc. $^{b}$ & -0.0025 & 20.721 & 24.2 & 16.1 \\
expt. & $-0.0018(5)$ & $20.754(3)$ & $23.0(5)$ & $17.5(5)$ \\
\hline 11,12$]$ & & & & \\
\hline
\end{tabular}

${ }^{a}$ Calculations of $g$ factor and hyperfine structure constant based on only the crystal-field contributions and those of superhyperfine parameters by directly fitting the unpaired spin densities in the previous work [13].

${ }^{b}$ Calculations of $g$ factor and hyperfine structure constant based on inclusion of both the crystal-field and charge transfer contributions and those of superhyperfine parameters based on the uniform formulae in this work.

clarify importance of the charge transfer contributions, the theoretical $g$ factor and hyperfine structure constant containing merely the crystal-field contributions are obtained and shown in Table II. The superhyperfine parameters (calc. ${ }^{a}$ ) obtained by directly fitting the unpaired spin densities in the previous work [13] are also collected in Table II.

\section{Discussion}

Table II reveals that the EPR parameters (calc. ${ }^{b}$ ) containing both the crystal-field and charge transfer contributions for $\mathrm{KMgF}_{3}: \mathrm{Cr}^{+}$are in reasonable agreement with the experimental data, while those (calc. ${ }^{a}$ ) for the $g$-shifts and the hyperfine structure constants including only the crystal-field contributions are not. This means that the improved $g$ and $A$ formulae involving both the crystal-field and charge transfer contributions can be regarded as more suitable and should be adopted here. Meanwhile, the superhyperfine parameters based on the unpaired spin densities determined from the uniform theoretical model and formulae in this work are also superior to those acquired from directly fitting the experimental superhyperfine parameters in the previous work [13].

1) The charge transfer mechanism involved here is the metal to ligand charge transfer arising from the low valence state and negativity of $\mathrm{Cr}^{+}$, instead of the conventional ligand to metal charge transfer [15]. As for the $g$-shift, the charge transfer contribution is the same (negative) in sign and much (60\%) larger in magnitude than the crystal-field one and should be taken into account in order to achieve more exact EPR studies for $\mathrm{KMgF}_{3}: \mathrm{Cr}^{+}$. Importantly, the $\Delta g$ is relatively small $\left(\approx 10^{-3}\right)$ for $3 d^{5}$ ions in octahedra (see Table II and Eq. (4)), and omission of the charge transfer contribution would inevitably lead to the much smaller magnitude of the resultant $\Delta g$ $\left(\right.$ calc. $\left.^{a}\right)$. Although the system exhibits weak covalency, the quantities $k_{\mathrm{CT}}^{\prime}$ and $\zeta_{\mathrm{CT}}^{\prime}$ for the charge transfer mechanism are comparable with but smaller than those of the crystal-field ones, since $\zeta_{p}^{0}\left(\approx 220 \mathrm{~cm}^{-1}\right.$ for $\left.\mathrm{F}^{-}\right)$is very close to $\zeta_{d}^{0}\left(\approx 230 \mathrm{~cm}^{-1}\right)$ for $\mathrm{Cr}^{+}$. So, for $3 d^{5}$ ions in fluorides with comparable ligand spin-orbit coupling interactions, the charge transfer contribution may be significant (corresponding to the low charge transfer level $E_{n}$ ) and thus bring forward obvious influence on $\Delta g$. It is noted that the conventional argument that the charge transfer contributions to zero-field splittings are negligible for $3 d^{5}$ ions under distorted fluorides is no longer suitable for $\Delta g$ analysis of $\mathrm{KMgF}_{3}: \mathrm{Cr}^{+}$due to the dominant second-order charge transfer perturbation term (see Eq. (4)).

2) As regards hyperfine structure constant, $A_{\mathrm{CT}}$ from the charge transfer contribution is the same (positive) in sign and much smaller (characterized by the relative ratio $A_{\mathrm{CT}} / A_{\mathrm{CF}} \approx 4 \%$ ) in magnitude as compared to $A_{\mathrm{CF}}$ from the crystal-field one, suggesting that the hyperfine structure constant is insensitive to the charge transfer contribution. This can be illustrated by the fact that hyperfine structure constant originates mainly from the isotropic contribution related to the core polarization constant $\kappa$ and only depends weakly upon the charge transfer contribution. Even though, inclusion of the charge transfer contribution could still induce some improvement in the theoretical hyperfine structure constant.

3) The present theoretical superhyperfine parameters based on the uniform model and formulae (with the unpaired spin densities quantitatively determined from the relevant molecular orbital coefficients) are in reasonable agreement with the experimental data. Furthermore, the unpaired spin densities $f_{s} \approx 0.59 \%$ and $f_{\sigma}-f_{\pi} \approx-0.12 \%$ obtained from the present calculations are not far from those $(\approx 0.61 \%$ and $-1.3 \%)$ based on directly fitting the experimental superhyperfine parameters of the previous works [13], only $f_{\sigma}-f_{\pi}$ of this work is smaller in magnitude than the previous fitting value. Agreement between the previous theoretical results (calc. ${ }^{a}$ ) and the observed values may be ascribed to the fact that the calculation errors happen to cancel one another during fitting procedure to the experimental superhyperfine parameters in the previous work [13]. Based on the uniform quantitative relationships between the unpaired spin densities and the related molecular orbital coefficients from the cluster approach, the present treatments can be regarded as more applicable than the previous studies [13].

\section{Conclusion}

The $g$ factor and hyperfine structure constant for $\mathrm{KMgF}_{3}: \mathrm{Cr}^{+}$are theoretically studied from the perturbation formulae containing both the crystal-field and metal to ligand charge transfer contributions from the cluster approach in a uniform way. The charge transfer contribution to $\Delta g$ is the same (negative) in sign and much larger in magnitude as compared to the crystal-field one. The conventional argument that the charge transfer contributions to zero-field splittings are negligible for $3 d^{5}$ ions in distorted fluorides is no longer suitable for $\Delta g$ analysis of $\mathrm{KMgF}_{3}: \mathrm{Cr}^{+}$here due to the dominant second-order 
charge transfer perturbation term. The charge transfer contribution to hyperfine structure constant exhibits the same sign and about $4 \%$ of the crystal-field one. The unpaired spin densities of the fluorine $2 s, 2 p \sigma$, and $2 p \pi$ orbitals are quantitatively acquired from the uniform relationships of the relevant molecular orbital coefficients, superior to the previous treatments by directly fitting the experimental superhyperfine parameters.

\section{Acknowledgments}

This work was supported by the Sichuan Province Academic and Technical Leaders Support Fund and Fundamental Research Funds for the Central Universities under grant No. ZYGX2012YB018.

\section{References}

[1] D.R. Lee, T.P.J. Han, B. Henderson, Appl. Phys. A 59, 365 (1994).

[2] G. Kitis, C. Furetta, C. Sanipoli, A. Scacco, Radiat. Prot. Dosim. 65, 93 (1996).

[3] A. Caramanian, J.P. Souron, P. Gredin, A. de Kozak, J. Derouet, B. Viana, J. Lumin. 104, 161 (2003).

[4] R. Augusto, Z. López, G.S. Dixon, Phys. Lett. A 58, 267 (1976).

[5] P. García-Fernández, A. Trueba, B. Gacía-Cueto, J.A. Aramburu, M.T. Barriuso, M. Moreno, Phys. Rev. B 83, 125123 (2011).

[6] A. Buzulutskov, Y. Vanyushkin, Nucl. Instrum. Methods Phys. Res. A 343, 241 (1994).

[7] A.S. Bhalla, R. Guo, R. Roy, Mater. Res. Innov. 4, $3(2000)$.

[8] A. Abragam, B. Bleaney, Electron Parmagnetic Resonance of Transition Ions, Oxford University Press, London 1970.

[9] A.S. Chakravarty, Introduction to the Magnetic Properties of Solids, Wiley InterScience, New York 1980.

[10] H.L. Van Camp, Y.W. Kim, Phys. Rev. B 11, 3098 (1975).

[11] J.J. Davies, K. Horai, J. Phys. C 4, 671 (1971).

[12] J.J. Davies, Phys. Lett. A 40, 423 (1972).

[13] T.P.P. Hall, W. Hayes, R.W.H. Stevenson, J. Chem. Phys. 38, 1977 (1963).

[14] A.B.P. Lever, Inorganic Electronic Spectroscopy, Elsevier, Amsterdam 1984.

[15] R.R. Sharma, in: Advances in Mössbauer Spectroscopy, Eds. B.V. Thosar, P.K. Lyenga, Elsevier Science, Amsterdam 1983.

[16] W.L. Yu, M.G. Zhao, Phys. Rev. B 37, 9254 (1988).

[17] S.Y. Wu, X.Y. Gao, H.N. Dong, J. Magn. Magn. Mater. 301, 67 (2006).

[18] H. Ziegler, Phys. Status Solidi B 49, 367 (1972).

[19] J.S. Griffith, The Theory of Transition-Metal Ions, Cambridge Press, London 1964.

[20] J. Owen, J.H.M. Thornley, Rep. Prog. Phys. 29, 675 (1966).

[21] T.P.P. Hall, W. Hayes, R.W.H. Stevenson, J. Wilkens, J. Chem. Phys. 39, 35 (1963).
[22] R.C. Weast, CRC Handbook of Chemistry and Physics, CRC Press, Boca Raton 1989.

[23] G. Fernandez Rodrigo, L. Pueyo, M. Moreno, M.T. Barriuso, J. Solid State Chem. 67, 64 (1987).

[24] M.G. Zhao, J.A. Xu, G.R. Bai, H.S. Xie, Phys. Rev. B 27, 1516 (1983).

[25] H. Watanabe, J. Phys. Chem. Solids 28, 961 (1967).

[26] E. Clementi, D.L. Raimondi, J. Chem. Phys. 38, 2686 (1963).

[27] E. Clementi, D.L. Raimondi, W.P. Reinhardt, J. Chem. Phys. 47, 1300 (1967).

[28] B.R. McGarvey, J. Phys. Chem. 71, 51 (1967).

[29] G.L. McPerson, R.C. Koch, G.D. Stucky, J. Chem Phys. 60, 1424 (1974).

[30] U. Kaufmann, A. Räuber, J. Schneider, J. Phys. C 8, L381 (1975). 